\title{
From the Editors and the Editorial Board
}

Our congratulations and best wishes to Alex Mueller and to his whole family!

We are proud to have Alex as a Member of our Board. We are very glad that during the last several years, Alex published his papers in our Journal [1-4]. These papers are very interesting for our readers and for the whole scientific community. Based on these papers, one can see that Alex Mueller is in excellent shape and one can expect a number of his deep insights and new papers in the near future.

\section{References}

1. Weyeneth, S., Mueller, K.: Oxygen isotope effect in cuprates results from polaron-induced superconductivity. J. Supercond. Novel Magn. 24, 1235 (2011)

2. Mueller, K.: Enhancing $\mathrm{T}_{\mathrm{c}}$ by Oxygen isotope effect in cuprates. J. Supercond. Novel Magn. 25, 2101 (2012)

3. Mueller, K., Shengelaya, A.: Dielectricly enhanced $T_{c}$ in underdoped cuprates. J. Supercond. Novel Magn. 26, 486 (2013)

4. Mueller, K.: The unique properties of superconductivity in cuprates. J. Supercond. Novel Magn. 27, 2163 (2014) 